\title{
Galerkin method for the scattering problem of strip gratings
}

\author{
Enxi Zheng ${ }^{1 *}$ (D) and Yujie Wang
}

${ }^{\text {*Correspondence: }}$

enxizheng2003@dlmu.edu.cn

'School of Science, Dalian Maritime

University, Dalian, P.R. China

\begin{abstract}
In this paper, the diffraction problem of periodic strip gratings is considered. The previous study of this problem usually concentrated on the numerical method; however, we try to analyze this problem and the convergence of the numerical solution from the mathematical point of view in this work. By use of the Dirichlet to Neumann operator on the slit between two strips, we reformulate the problem to an operator equation. The well-posedness of the solution to the operator equation is proved. The Galerkin method is applied to solve this operator equation and the convergence result of the numerical solution is also derived. Finally, some numerical experiments are presented to show the effectiveness of our method and verify the theoretical convergence result.
\end{abstract}

Keywords: Strip grating; Dirichlet to Neumann map; Helmholtz equation; Galerkin method

\section{Introduction}

The scattering theory of periodic structures has a wide variety of applications, for example, the micro-optics and the antenna engineering. In optics, the periodic structure is also called diffraction gratings. Introduction to the problem of electromagnetic diffraction through periodic structures and the corresponding numerical methods can be found in [1]. The reviews on the diffractive optics technology and the mathematical analysis of diffraction gratings are presented in [2] and [3], respectively.

In this paper, we focus on the diffraction problem of periodic perfectly conducting strip gratings. This is a classic model which has been investigated by many researchers. In [4] the method of moments (MoM) is employed to analyze the diffraction problem of strip gratings located in free space. In papers $[5,6]$ the same numerical method is used to solve the strip grating problem where the strips are printed on a dielectric substrate. As to the improved MoM for scattering problem of periodic strip gratings, we refer to paper [7] and the references therein. In addition to the MoM, there are also many other numerical methods for the strip gratings problem. For example, the singular integral equation approach is proposed to deal with the plane wave diffraction by an infinite strip grating at oblique incidence in [8]. Fourier modal method (FMM), also called combined boundary conditions method (CBCM), is introduced in [9-11]. CBCM is applied for solving finite strip grating problem in [11]. Because of the slow convergence and Gibbs phenomenon at the tips of the strips, $\mathrm{CBCM}$ has been substantially improved in [12] with the aid of adaptive spatial resolution in [11]. The parametric formulation of CBCM in [12] improves the

(c) The Author(s) 2019. This article is distributed under the terms of the Creative Commons Attribution 4.0 International License (http://creativecommons.org/licenses/by/4.0/), which permits unrestricted use, distribution, and reproduction in any medium, provided you give appropriate credit to the original author(s) and the source, provide a link to the Creative Commons license, and indicate if changes were made. 
convergence rate, the computational efficiency, and the numerical accuracy. In [13] the authors employ this improved method in multilayered structures of strip gratings. More references about $\mathrm{CBCM}$ can be found in [14]. In mathematics, because the grating diffraction problem is governed by differential equation, the finite difference method (FDM) and the finite element method (FEM) can also be applied in solving the problem. In the numerical experiment part of this paper, we compare our method with the FDM.

The studies above concentrate on the mathematical model and the numerical method of the strip gratings. In this paper, we will reformulate the diffraction problem of strip grating into an operator equation by use of the Dirichlet to Neumann (DtN) operator. Then we will give a rigorous mathematical analysis of the solution and the Galerkin method which is used to solve the operator equation. The convergence of the Galerkin method has also been verified by the numerical experiments.

The paper is organized as follows. In Sect. 2, we give some notations for describing the strip gratings problem and reformulate this problem into an operator equation by use of DtN operator on the slit between two strips. In Sect. 3, the well-posedness of the solution to the operator equation derived in Sect. 2 is proved. The well-posedness contains the existence, the uniqueness, and the stability of the solution. In Sect. 4, the Galerkin method is employed to solve the operator equation. The uniqueness and the convergence of the numerical solution are proved. We also obtain the error estimate of the numerical solution in Theorem 5. The concrete computing process is given at the end of this section. In Sect. 5, some numerical experiments are presented to show the effectiveness of the Galerkin method and convergence order proved in Sect. 4 is also verified by numerical Example 1 in this section.

\section{Formulation of the problem}

In this section, some notations are firstly presented to help us describe the diffraction problem of periodic strip gratings with period $d$. Then the definition of quasi-periodic, the famous Rayleigh expansion of diffraction field, and the DtN operator are introduced. Finally, we reformulate the diffraction problem into an operator equation through simple calculation and the knowledge prepared above.

Assume that the length of each slit is $L(L<d)$, and the slits are separated by perfect conductive material strips $\tilde{\Gamma}$, see Figure 1 . Denote the slit and the perfect conductive strip in one period by $\Gamma_{0}$ and $\Gamma_{1}$, respectively:

$$
\begin{aligned}
& \Gamma_{0}=\left\{\left(x_{1}, x_{2}\right) \in \mathbb{R}^{2} ; 0<x_{1}<L, x_{2}=0\right\}, \\
& \Gamma_{1}=\left\{\left(x_{1}, x_{2}\right) \in \mathbb{R}^{2} ; L<x_{1}<d, x_{2}=0\right\} .
\end{aligned}
$$

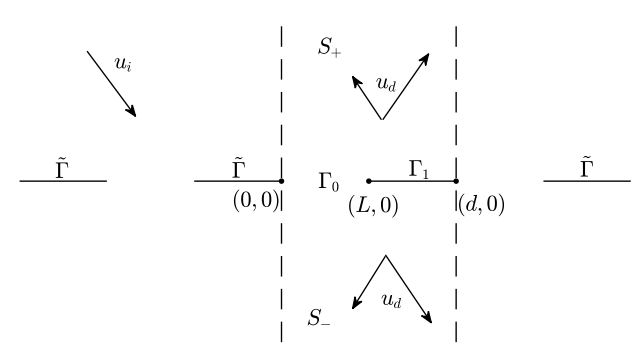

Figure 1 The diffraction problem of strip grating 
Furthermore, define $\Gamma$ as follows:

$$
\Gamma=\left\{\left(x_{1}, x_{2}\right) \in \mathbb{R}^{2} ; 0<x_{1}<d, x_{2}=0\right\} .
$$

Suppose that the domain

$$
S=\left\{\left(x_{1}, x_{2}\right) \in \mathbb{R}^{2} ; 0<x_{1}<d,\left(x_{1}, x_{2}\right) \notin \Gamma_{1}\right\}
$$

is filled with a homogeneous medium. Let $S_{+}$and $S_{-}$denote the domain upon and below the strip gratings, respectively:

$$
\begin{aligned}
& S_{+}=\left\{\left(x_{1}, x_{2}\right) \in \mathbb{R}^{2} ; 0<x_{1}<d, x_{2}>0\right\}, \\
& S_{-}=\left\{\left(x_{1}, x_{2}\right) \in \mathbb{R}^{2} ; 0<x_{1}<d, x_{2}<0\right\} .
\end{aligned}
$$

Assume that the plane wave $u_{i}=e^{i \alpha x_{1}-i \beta x_{2}}$ is the incident wave upon the grating, where $\alpha=$ $k \sin \theta, \beta=k \cos \theta, k>0$ is the wave number, and $\theta \in(-\pi / 2, \pi / 2)$ is the angle of incidence. Denote the diffracted field by $u_{d}$. Then the total field $u_{t}$ is

$$
u_{t}= \begin{cases}u_{i}+u_{d}, & x_{2}>0, \\ u_{d}, & x_{2}<0,\end{cases}
$$

and the diffraction problem of strip gratings reads as follows: when the incident field $u_{i}$ is given, find the total field $u_{t}$ such that

$$
\begin{aligned}
& \Delta u_{t}+k^{2} u_{t}=0, \quad \text { in } S, \\
& u_{t}=0, \quad \text { on } \Gamma_{1} .
\end{aligned}
$$

Among all the solutions of equations (2) and (3), we are interested in the quasi-periodic solution, i.e., $u_{t} e^{-i \alpha x}$ is a periodic function in $x_{1}$ with period $d$. Moreover, we require the diffracted field $u_{d}$ to satisfy the bounded outgoing wave condition in $S_{+}$and $S_{-}$.

In domains $S_{+}$and $S_{-}$, the famous Rayleigh expansion of $u_{d}$ is

$$
\begin{array}{ll}
u_{d}\left(x_{1}, x_{2}\right)=\sum_{n=-\infty}^{\infty} a_{n} e^{i\left(\alpha_{n}+\alpha\right) x_{1}+i \beta_{n} x_{2}}, & \left(x_{1}, x_{2}\right) \in S_{+}, \\
u_{d}\left(x_{1}, x_{2}\right)=\sum_{n=-\infty}^{\infty} b_{n} e^{i\left(\alpha_{n}+\alpha\right) x_{1}-i \beta_{n} x_{2}}, & \left(x_{1}, x_{2}\right) \in S_{-},
\end{array}
$$

where $\alpha_{n}=2 \pi n / d$,

$$
\beta_{n}= \begin{cases}\left(k^{2}-\left(\alpha_{n}+\alpha\right)^{2}\right)^{1 / 2}, & n \in A, \\ i\left(\left(\alpha_{n}+\alpha\right)^{2}-k^{2}\right)^{1 / 2}, & n \notin A,\end{cases}
$$

$A=\left\{n \in \mathbb{Z}, k^{2}-\left(\alpha_{n}+\alpha\right)^{2}>0\right\}$ and $a_{n}, b_{n}$ are coefficients to be determined. We further assume that $k \neq\left|\alpha_{n}+\alpha\right|$ for every $n \in \mathbb{Z}$ in order to avoid resonances. Define

$$
u_{d}^{+}\left(x_{1}\right)=\lim _{x_{2} \rightarrow 0_{+}} u_{d}\left(x_{1}, x_{2}\right), \quad u_{d}^{-}\left(x_{1}\right)=\lim _{x_{2} \rightarrow 0_{-}} u_{d}\left(x_{1}, x_{2}\right)
$$




$$
\frac{\partial u_{d}^{+}}{\partial x_{2}}\left(x_{1}\right)=\lim _{x_{2} \rightarrow 0_{+}} \frac{\partial u_{d}}{\partial x_{2}}\left(x_{1}, x_{2}\right), \quad \frac{\partial u_{d}^{-}}{\partial x_{2}}\left(x_{1}\right)=\lim _{x_{2} \rightarrow 0_{-}} \frac{\partial u_{d}}{\partial x_{2}}\left(x_{1}, x_{2}\right),
$$

and $u_{i}^{+}\left(x_{1}\right), \frac{\partial u_{t}^{-}}{\partial x_{2}}\left(x_{1}\right)$, etc. are defined in the same way. Then we have the following integral expression of $a_{n}$ and $b_{n}$ :

$$
\begin{aligned}
& a_{n}=\frac{1}{d} \int_{0}^{d} u_{d}^{+}\left(x_{1}\right) e^{-i\left(\alpha_{n}+\alpha\right) x_{1}} \mathrm{~d} x_{1}, \\
& b_{n}=\frac{1}{d} \int_{0}^{d} u_{d}^{-}\left(x_{1}\right) e^{-i\left(\alpha_{n}+\alpha\right) x_{1}} \mathrm{~d} x_{1} .
\end{aligned}
$$

For any quasi-periodic function $f\left(x_{1}\right)$, i.e., $f\left(x_{1}\right)$ has the following expansion:

$$
f\left(x_{1}\right)=\sum_{n=-\infty}^{+\infty} f_{n} e^{i\left(\alpha_{n}+\alpha\right) x_{1}}, \quad \text { where } f_{n}=\frac{1}{d} \int_{0}^{d} f\left(x_{1}\right) e^{-i\left(\alpha_{n}+\alpha\right) x_{1}} \mathrm{~d} x_{1}
$$

define the Dirichlet to Neumann operator $T_{1}$ and $T_{2}$ as follows:

$$
\begin{aligned}
& T_{1} f\left(x_{1}\right)=\sum_{n=-\infty}^{+\infty} i \beta_{n} f_{n} e^{i\left(\alpha_{n}+\alpha\right) x_{1}}, \\
& T_{2} f\left(x_{1}\right)=\sum_{n=-\infty}^{+\infty}-i \beta_{n} f_{n} e^{i\left(\alpha_{n}+\alpha\right) x_{1}} .
\end{aligned}
$$

By simple calculation, we can get

$$
\begin{aligned}
& \frac{\partial u_{d}^{+}}{\partial x_{2}}\left(x_{1}\right)=T_{1}\left(u_{d}^{+}\right)\left(x_{1}\right), \quad 0<x_{1}<d, \\
& \frac{\partial u_{d}^{-}}{\partial x_{2}}\left(x_{1}\right)=T_{2}\left(u_{d}^{-}\right)\left(x_{1}\right), \quad 0<x_{1}<d .
\end{aligned}
$$

Since the total field $u_{t}$ and the normal derivative $\frac{\partial u_{t}}{\partial x_{2}}$ are continuous across $\Gamma_{1}$ and $u_{t}=0$ on $\Gamma_{0}$,

$$
\begin{aligned}
& u_{t}^{+}\left(x_{1}\right)=u_{t}^{-}\left(x_{1}\right), \quad 0<x_{1}<d, \\
& \frac{\partial u_{t}^{+}}{\partial x_{2}}\left(x_{1}\right)=\frac{\partial u_{t}^{-}}{\partial x_{2}}\left(x_{1}\right), \quad 0<x_{1}<L .
\end{aligned}
$$

Let $u\left(x_{1}\right)=u_{d}^{-}\left(x_{1}\right), x_{1} \in(0, L)$, then

$$
u_{d}^{-}\left(x_{1}\right)=E_{0}(u), \quad x_{1} \in(0, d)
$$

where $E_{0}$ is the zero extension operator defined by the following:

$$
E_{0}(u)= \begin{cases}u\left(x_{1}\right), & 0<x_{1}<L \\ 0, & L \leq x_{1} \leq d\end{cases}
$$

From (1), (6)-(9), and (10) we obtain

$$
u_{i}^{+}\left(x_{1}\right)+u_{d}^{+}\left(x_{1}\right)=E_{0}(u)\left(x_{1}\right), \quad x_{1} \in(0, d),
$$




$$
\frac{\partial u_{i}^{+}}{\partial x_{2}}\left(x_{1}\right)+T_{1}\left(u_{d}^{+}\right)=T_{2}\left(u_{d}^{-}\right), \quad x_{1} \in(0, L)
$$

Substitute (10) and (11) into (12)

$$
\frac{\partial u_{i}^{+}}{\partial x_{2}}\left(x_{1}\right)+T_{1}\left(E_{0}(u)-u_{i}^{+}\right)=T_{2}\left(E_{0}(u)\right), \quad x_{1} \in(0, L) .
$$

From the definitions of $T_{1}$ and $T_{2}$, we know that

$$
\begin{aligned}
& T_{1}\left(u_{i}^{+}\right)=i \beta e^{i \alpha x_{1}}, \quad x_{1} \in(0, L), \\
& T_{1}\left(E_{0}(u)\right)=-T_{2}\left(E_{0}(u)\right), \quad x_{1} \in(0, L) .
\end{aligned}
$$

Substitute (14) and (15) into (13)

$$
T_{2}\left(E_{0}(u)\right)=-i \beta e^{i \alpha x_{1}}, \quad x_{1} \in(0, L)
$$

Denote $T=T_{2} \circ E_{0}$ and $g\left(x_{1}\right)=-i \beta e^{i \alpha x_{1}}$, then equation (16) can be rewritten to

$$
T u=g, \quad x_{1} \in(0, L) .
$$

Thus the diffraction problem of strip gratings can be reformulated into the operator equation (17). Once the solution of (17) is derived, the diffraction field $u_{d}$ and the total field $u_{t}$ can be obtained through (4), (5), and (1).

\section{Well-posedness analysis}

In this section, we will prove the well-posedness of the solution to (17). In order to give the uniqueness and existence of the solution to (17), we introduce the following definitions of spaces and norms.

For any real number $s$, define Sobolev spaces

$$
\begin{aligned}
& H_{\alpha}^{s}(\Gamma)=\left\{u \in H^{s}(\Gamma) ; u\left(x_{1}\right) e^{-i \alpha x_{1}} \text { is periodic in } x_{1} \text { with period } d\right\} \\
& H_{\alpha}^{s}\left(\Gamma_{0}\right)=\left\{u \in\left(C_{0}^{\infty}\left(\Gamma_{0}\right)\right)^{\prime}, u=\left.U\right|_{\Gamma_{0}} \text {, for some } U \in H_{\alpha}^{s}(\Gamma)\right\} \\
& H_{\alpha, *}^{s}\left(\Gamma_{0}\right)=\left\{u \in\left(C_{0}^{\infty}\left(\Gamma_{0}\right)\right)^{\prime}, E_{0}(u) \in H_{\alpha}^{s}(\Gamma)\right\}
\end{aligned}
$$

with norms

$$
\begin{aligned}
& \|u\|_{s, \Gamma}=\left(d \sum_{n=-\infty}^{+\infty}\left(1+\left(\alpha_{n}+\alpha\right)^{2}\right)^{s}\left|u_{n}\right|^{2}\right)^{\frac{1}{2}}, \quad u \in H_{\alpha}^{s}(\Gamma), \\
& \|u\|_{s, \Gamma_{0}}=\inf _{U \in H_{\alpha}^{s}(\Gamma),\left.U\right|_{\Gamma_{0}}=u}\|U\|_{s, \Gamma}, \quad u \in H_{\alpha}^{s}\left(\Gamma_{0}\right), \\
& \|u\|_{s, *, \Gamma_{0}}=\left\|E_{0}(u)\right\|_{s, \Gamma}, \quad u \in H_{\alpha, *}^{s}\left(\Gamma_{0}\right),
\end{aligned}
$$

where

$$
u_{n}=\frac{1}{d} \int_{0}^{d} u\left(x_{1}\right) e^{-i\left(\alpha_{n}+\alpha\right) x_{1}} \mathrm{~d} x_{1}
$$


Using the above notations, the operator equation problem (17) can be formulated as follows: given $g \in H_{\alpha}^{-\frac{1}{2}}\left(\Gamma_{0}\right)$, find $u \in H_{\alpha, *}^{\frac{1}{2}}(\Gamma)$ such that equation (17) is satisfied.

In the following, we will present two lemmas about the properties of spaces $H_{*}^{\frac{1}{2}}\left(\Gamma_{0}\right)$, $H_{*}^{-\frac{1}{2}}\left(\Gamma_{1}\right)$ and the operator $T$. With the aid of these two lemmas, we can obtain the wellposedness of the solution to equation (17).

Lemma 1 The operator $T: H_{\alpha, *}^{\frac{1}{2}}\left(\Gamma_{0}\right) \rightarrow H_{\alpha}^{-\frac{1}{2}}\left(\Gamma_{0}\right)$ is a linear bounded operator.

Proof Denote $u_{n}=\frac{1}{d} \int_{0}^{d} E_{0}(u) e^{-i\left(\alpha_{n}+\alpha\right) x_{1}} \mathrm{~d} x_{1}$ for $u \in H_{\alpha, *}^{\frac{1}{2}}\left(\Gamma_{0}\right)$, and define

$$
V\left(x_{1}\right)=\sum_{n=-\infty}^{+\infty}-i \beta_{n} u_{n} e^{i\left(\alpha_{n}+\alpha\right) x_{1}}, \quad x_{1} \in(0, d)
$$

Then $\left.V\right|_{\Gamma_{0}}=T u$. From the definition of $\|\cdot\|_{-\frac{1}{2}, \Gamma_{0}}$, we have

$$
\begin{aligned}
\|T u\|_{-\frac{1}{2}, \Gamma_{0}} & =\inf _{U \in H_{\alpha}^{s}(\Gamma),\left.U\right|_{\Gamma_{0}}=T u}\|U\|_{-\frac{1}{2}, \Gamma} \leq\|V\|_{-\frac{1}{2}, \Gamma} \\
& =\left(\sum_{n=-\infty}^{+\infty}\left[1+\left(\alpha_{n}+\alpha\right)\right]^{-\frac{1}{2}}\left|\beta_{n}\right|^{2}\left|u_{n}\right|^{2}\right)^{\frac{1}{2}} \\
& \leq\left(\sum_{n=-\infty}^{+\infty}\left[1+\left(\alpha_{n}+\alpha\right)\right]^{-\frac{1}{2}}\left[k^{2}+\left(\alpha_{n}+\alpha\right)^{2}\right]\left|u_{n}\right|^{2}\right)^{\frac{1}{2}} \\
& \leq C_{k}\left(\sum_{n=-\infty}^{+\infty}\left[1+\left(\alpha_{n}+\alpha\right)\right]^{\frac{1}{2}}\left|u_{n}\right|^{2}\right)^{\frac{1}{2}} \\
& =C_{k}\|u\|_{\frac{1}{2}, *, \Gamma_{0}},
\end{aligned}
$$

where $C_{k}=\max \{k, 1\}^{\frac{1}{2}}$.

Lemma 2 The embedding operator $I_{*}: H_{\alpha, *}^{\frac{1}{2}}\left(\Gamma_{0}\right) \rightarrow H_{\alpha}^{-\frac{1}{2}}\left(\Gamma_{0}\right)$ is a compact operator.

Proof The operator $I_{*}$ can be decomposed into $I_{*}=R \circ I \circ E_{0}$, where $E_{0}$ is the zero extension operator from $H_{\alpha, *}^{\frac{1}{2}}\left(\Gamma_{0}\right)$ to $H_{\alpha}^{\frac{1}{2}}(\Gamma), I$ is the embedding operator from $H_{\alpha}^{\frac{1}{2}}(\Gamma)$ to $H_{\alpha}^{-\frac{1}{2}}(\Gamma)$, and $R$ is the restriction operator from $H_{\alpha}^{-\frac{1}{2}}(\Gamma)$ to $H_{\alpha}^{-\frac{1}{2}}\left(\Gamma_{0}\right)$. Since $E_{0}$ and $R$ are bounded and $I$ is compact, we obtain that $I_{*}$ is compact.

Theorem 1 Assume that $\beta_{n} \neq 0$ for all $n \in \mathbb{Z}$, then the homogeneous equation

$$
T u=0, \quad u \in H_{\alpha, *}^{\frac{1}{2}}\left(\Gamma_{0}\right)
$$

has only one solution $u=0$.

Proof From $T u=0$, we have $\langle T u, u\rangle=0$. Furthermore,

$$
\langle T u, u\rangle=\int_{\Gamma_{0}} T u \bar{u} \mathrm{~d} x_{1}=\int_{\Gamma} T u \overline{E_{0}(u)} \mathrm{d} x_{1}
$$




$$
\begin{aligned}
& =\int_{\Gamma} \sum_{n=-\infty}^{+\infty}-i \beta_{n} u_{n} e^{i\left(\alpha_{n}+\alpha\right) x_{1}} \overline{\sum_{n=-\infty}^{+\infty} u_{n} e^{i\left(\alpha_{n}+\alpha\right) x_{1}}} \mathrm{~d} x_{1} \\
& =-d \sum_{n=-\infty}^{+\infty} i \beta_{n}\left|u_{n}\right|^{2} \\
& =-d \sum_{n \in A} i \beta_{n}\left|u_{n}\right|^{2}-d \sum_{n \notin A} i \beta_{n}\left|u_{n}\right|^{2} .
\end{aligned}
$$

From $\langle T u, u\rangle=0$, we can get

$$
\begin{aligned}
& \operatorname{Re}\langle T u, u\rangle=-d \sum_{n \notin A} i \beta_{n}\left|u_{n}\right|^{2}=0, \\
& \operatorname{Im}\langle T u, u\rangle=-d \sum_{n \in A} \beta_{n}\left|u_{n}\right|^{2}=0 .
\end{aligned}
$$

Since $\beta_{n} \neq 0$ for all $n \in \mathbb{Z}$, from the above two equations, we can deduce that $u_{n}=0$ for all $n \in \mathbb{Z}$. Thus $E_{0}(u)=0$, i.e., $u=0$.

Theorem 2 Assume that $\beta_{n} \neq 0$ for all $n \in \mathbb{Z}$. Then, for any $g \in H_{\alpha}^{-\frac{1}{2}}\left(\Gamma_{0}\right)$, the operator equation $T u=g$ has a unique solution $u \in H_{\alpha, *}^{\frac{1}{2}}\left(\Gamma_{0}\right)$, and

$$
\|u\|_{\frac{1}{2}, *, \Gamma_{0}} \leq C\|g\|_{-\frac{1}{2}, \Gamma_{0}}
$$

where $C>0$ is a constant independent of $g$.

Proof Define operator $B: H_{\alpha, *}^{\frac{1}{2}}\left(\Gamma_{0}\right) \rightarrow H_{\alpha}^{-\frac{1}{2}}\left(\Gamma_{0}\right)$ and the corresponding bilinear form $b(\cdot, \cdot)$ as follows:

$$
B=T+\sqrt{2} k I_{*}, \quad b(u, v)=\langle B u, v\rangle .
$$

Since the operators $T$ and $I_{*}$ are bounded, $b(\cdot, \cdot)$ is a bounded bilinear form on $H_{\alpha, *}^{\frac{1}{2}}\left(\Gamma_{0}\right) \times$ $H_{\alpha, *}^{\frac{1}{2}}\left(\Gamma_{0}\right)$. Next, we will show that $b(\cdot, \cdot)$ has a lower bound. By simple calculation, we can derive the following two inequalities:

$$
\begin{aligned}
& |b(u, v)| \geq \frac{\sqrt{2}}{2}(|\operatorname{Re}\{b(u, v)\}|+|\operatorname{Im}\{b(u, v)\}|), \\
& \sqrt{\left|\left(\alpha_{n}+\alpha\right)^{2}-k^{2}\right|} \geq \sqrt{\left|\left(\alpha_{n}+\alpha\right)^{2}+k^{2}\right|}-\sqrt{2} k .
\end{aligned}
$$

Thus

$$
\begin{aligned}
|b(u, v)| & =\left|\langle T u, v\rangle+\left\langle\sqrt{2} k I_{*} u, v\right\rangle\right| \\
& =\left.\left|\int_{\Gamma} T u \overline{E_{0}(u)} \mathrm{d} x_{1}+\sqrt{2} k \int_{\Gamma}\right| E_{0}(u)\right|^{2} \mathrm{~d} x_{1} \mid \\
& =\left.\left|d \sum_{n=-\infty}^{+\infty}-i \beta_{n}\right| u_{n}\right|^{2}+\sqrt{2} k d \sum_{n=-\infty}^{+\infty}\left|u_{n}\right|^{2} \mid
\end{aligned}
$$




$$
\begin{aligned}
= & \left.\left|d \sum_{n \in A}-i \sqrt{k^{2}-\left(\alpha_{n}+\alpha\right)^{2}}\right| u_{n}\right|^{2} \\
& +d \sum_{n \notin A} \sqrt{\left(\alpha_{n}+\alpha\right)^{2}-k^{2}}\left|u_{n}\right|^{2}+\sqrt{2} k d \sum_{n=-\infty}^{+\infty}\left|u_{n}\right|^{2} \mid \\
\geq & \frac{\sqrt{2}}{2}\left(\left.\left|d \sum_{n \in A} \sqrt{k^{2}-\left(\alpha_{n}+\alpha\right)^{2}}\right| u_{n}\right|^{2} \mid\right. \\
& \left.+\left.\left|d \sum_{n \notin A} \sqrt{\left(\alpha_{n}+\alpha\right)^{2}-k^{2}}\right| u_{n}\right|^{2}+\sqrt{2} k d \sum_{n=-\infty}^{+\infty}\left|u_{n}\right|^{2} \mid\right) \\
\geq & \frac{\sqrt{2}}{2}\left(d \sum_{n=-\infty}^{+\infty} \sqrt{\left(\alpha_{n}+\alpha\right)^{2}+k^{2}}\left|u_{n}\right|^{2}\right. \\
& \left.-d \sum_{n \in A} \sqrt{2} k\left|u_{n}\right|^{2}-d \sum_{n \notin A} \sqrt{2} k\left|u_{n}\right|^{2}+\sqrt{2} k d \sum_{n=-\infty}^{+\infty}\left|u_{n}\right|^{2}\right) \\
\geq & \frac{\sqrt{2}}{2}\left(d \sum_{n=-\infty}^{+\infty} \sqrt{\left(\alpha_{n}+\alpha\right)^{2}+k^{2}}\left|u_{n}\right|^{2}\right) \\
& \left.+\infty \alpha_{n=-\infty}^{+\infty}\right)
\end{aligned}
$$

where $C=\frac{\sqrt{2}}{2} \min \{1, k\}$. By Lax-Milgram theorem, the operator $B$ has a bounded inverse $B^{-1}: H_{\alpha}^{-\frac{1}{2}}\left(\Gamma_{0}\right) \rightarrow H_{\alpha, *}^{\frac{1}{2}}\left(\Gamma_{0}\right)$. Then the operator equation $T u=g$ can be rewritten as

$$
\left(B-\sqrt{2} k I_{*}\right) u=g
$$

From Theorem 1 , we know that $-\sqrt{2} k$ is not an eigenvalue of $B$. Because $I_{*}$ is a compact operator, by use of Fredholm alternative theorem, the operator equation $T u=g$ has a unique solution $u \in H_{\alpha, *}^{\frac{1}{2}}\left(\Gamma_{0}\right)$ and

$$
\|u\|_{\frac{1}{2}, *, \Gamma_{0}} \leq C\|g\|_{-\frac{1}{2}, \Gamma_{0}}
$$

where $C$ is a constant independent of $g$.

\section{Galerkin method}

In this section we introduce the Galerkin method to solve equation (17) numerically. Assume

$$
V_{N}=\operatorname{span}\left\{\varphi_{1}, \varphi_{2}, \ldots, \varphi_{N}\right\}
$$

where

$$
\varphi_{n}=\sin \left(\frac{n \pi x_{1}}{L}\right), \quad n=1,2, \ldots, N
$$

The Galerkin method to solve equation (17) is to find $u_{N} \in V_{N}$ such that

$$
\left\langle T u_{N}, \varphi_{m}\right\rangle=\left\langle g, \varphi_{m}\right\rangle, \quad m=1,2, \ldots, N
$$


where

$$
\begin{aligned}
& \left\langle T u_{N}, \varphi_{m}\right\rangle=\int_{0}^{L} T u_{N} \overline{\varphi_{m}} \mathrm{~d} x_{1}, \\
& \left\langle g, \varphi_{m}\right\rangle=\int_{0}^{L} g \overline{\varphi_{m}} \mathrm{~d} x_{1} .
\end{aligned}
$$

Theorem 3 The Galerkin equation (18) has a unique solution $u_{N} \in V_{N}$.

Proof For the finite dimensional problem (18), we only need to show that the homogeneous equation

$$
\left\langle T u_{N}, \varphi_{m}\right\rangle=0, \quad m=1,2, \ldots, N
$$

has only one solution $u_{N}=0$. From (19), we can get $\left\langle T u_{N}, u_{N}\right\rangle=0$. Thus the proof for $u_{N}=0$ is similar to Theorem 1 .

The next theorem follows from standard estimates for Galerkin method associated with compact operator equation, so we omit the proof here and refer to [15] for details.

Theorem 4 Assume that $\beta_{n} \neq 0$ for all $n \in \mathbb{Z}$, $u$ is the solution of (17), and $u_{N}$ is the solution of (18). When $N$ is large enough,

$$
\left\|u-u_{N}\right\|_{\frac{1}{2}, *, \Gamma_{0}} \leq C \inf _{v_{N} \in V_{N}}\left\|u-v_{N}\right\|_{\frac{1}{2}, *, \Gamma_{0}}
$$

where $C$ is a positive constant independent of $N$.

For $s \in \mathbb{R}$, define space $H_{p}^{s}\left(\Gamma_{0}\right)$ as follows:

$$
H_{p}^{s}\left(\Gamma_{0}\right)=\left\{u=\sum_{n=1}^{+\infty} a_{n} \sin \left(\frac{n \pi x}{L}\right) ; \frac{L}{2} \sum_{n=1}^{+\infty}\left[1+\left(\frac{n \pi}{L}\right)^{2}\right]^{s}\left|a_{n}\right|^{2}<+\infty\right\} .
$$

The norm in $H_{p}^{s}\left(\Gamma_{0}\right)$ is given by

$$
\|u\|_{s, p, \Gamma_{0}}=\left(\frac{L}{2} \sum_{n=1}^{+\infty}\left[1+\left(\frac{n \pi}{L}\right)^{2}\right]^{s}\left|a_{n}\right|^{2}\right)^{\frac{1}{2}} .
$$

With a little extension of Lemma 4.11 and Example 4.15 in [16] to quasi-periodic functions, the space $H_{\alpha, *}^{s}\left(\Gamma_{0}\right)$ is equal to $H_{p}^{s}\left(\Gamma_{0}\right)$, and the norms $\|\cdot\|_{s, *, \Gamma_{0}}$ and $\|\cdot\|_{s, p, \Gamma_{0}}$ are equivalent when $0<s<1$. Then the conclusion of Theorem 4 can be rewritten as

$$
\left\|u-u_{N}\right\|_{\frac{1}{2}, *, \Gamma_{0}} \leq C \inf _{\nu_{N} \in V_{N}}\left\|u-v_{N}\right\|_{\frac{1}{2}, p, \Gamma_{0}}
$$

or

$$
\left\|u-u_{N}\right\|_{\frac{1}{2}, p, \Gamma_{0}} \leq C \inf _{v_{N} \in V_{N}}\left\|u-v_{N}\right\|_{\frac{1}{2}, p, \Gamma_{0}} .
$$


Theorem 5 Assume that $\beta_{n} \neq 0$ for all $n \in \mathbb{Z}, u \in H_{p}^{s}\left(\Gamma_{0}\right)\left(s>\frac{1}{2}\right)$ is the solution of (17), and $u_{N}$ is the solution of (18), then when $N$ is large enough,

$$
\left\|u-u_{N}\right\|_{\frac{1}{2}, *, \Gamma_{0}} \leq \frac{C}{N^{s-\frac{1}{2}}}\|u\|_{s, p, \Gamma_{0}}
$$

or

$$
\left\|u-u_{N}\right\|_{\frac{1}{2}, p, \Gamma_{0}} \leq \frac{C}{N^{s-\frac{1}{2}}}\|u\|_{s, p, \Gamma_{0}}
$$

where $C$ is a positive constant independent of $N$.

Proof Because

$$
\begin{aligned}
\inf _{v_{N} \in V_{N}}\left\|u-v_{N}\right\|_{\frac{1}{2}, p, \Gamma_{0}} & \leq\left\|\sum_{n=1}^{+\infty} a_{n} \sin \left(\frac{n \pi x}{L}\right)-\sum_{n=1}^{N} a_{n} \sin \left(\frac{n \pi x}{L}\right)\right\|_{\frac{1}{2}, p, \Gamma_{0}} \\
& =\left(\frac{L}{2} \sum_{n=N+1}^{+\infty}\left(1+\left(\frac{n \pi}{L}\right)^{2}\right)^{\frac{1}{2}}\left|a_{n}\right|^{2}\right)^{\frac{1}{2}} \\
& \leq\left(\frac{L}{2} \sum_{n=N+1}^{+\infty}\left(1+\left(\frac{n \pi}{L}\right)^{2}\right)^{\frac{1}{2}-s}\left(1+\left(\frac{n \pi}{L}\right)^{2}\right)^{s}\left|a_{n}\right|^{2}\right)^{\frac{1}{2}} \\
& \leq\left(\frac{L}{2} \sum_{n=N+1}^{+\infty}\left(\frac{N \pi}{L}\right)^{1-2 s}\left(1+\left(\frac{n \pi}{L}\right)^{2}\right)^{s}\left|a_{n}\right|^{2}\right)^{\frac{1}{2}} \\
& \leq \frac{C}{N^{s-\frac{1}{2}}}\|u\|_{s, p, \Gamma_{0}},
\end{aligned}
$$

then (22) and (23) are derived by combining the above inequality with (20) and (21).

\section{Numerical results}

In this section we demonstrate the numerical results of our method. All computations are performed using MATLAB. In all the following examples, we set the period $d=4$, the length of the slit $L=2$, and $\Gamma_{0}=\left\{\left(x_{1}, 0\right) ; 0<x_{1}<2\right\}$.

Example 1 In this example we consider the convergence results of our Galerkin method. Let $u\left(x_{1}\right)=x_{1}\left(x_{1}-2\right), x_{1} \in(0,2)$ be the exact solution of the operator equation (17) with $k=1, \alpha=0$, and the corresponding right-hand side function

$$
g\left(x_{1}\right)=-2 \sum_{n=-\infty}^{+\infty} i \beta_{n} \frac{n \pi+(-1)^{n} n \pi+2 i-(-1)^{n} 2 i}{n^{3} \pi^{3}} e^{i \frac{n \pi x_{1}}{2}} .
$$

Moreover, $u\left(x_{1}\right)$ has the following expansion:

$$
u\left(x_{1}\right)=x_{1}\left(x_{1}-2\right)=\sum_{n=1}^{+\infty} \frac{32}{(2 n-1)^{3} \pi^{3}} \sin \left(\frac{(2 n-1) \pi x}{2}\right), \quad x \in(0,2)
$$


Table $1\left\|u-u_{N}\right\|_{\frac{1}{2}, p, \Gamma_{0}}$ and $\left\|u-u_{N}\right\|_{\frac{1}{2}, *, \Gamma_{0}}$ with respect to $N$

\begin{tabular}{lllllll}
\hline$N$ & 2 & 4 & 8 & 16 & 32 & 64 \\
\hline$\left\|u-u_{N}\right\|_{\frac{1}{2}, p, \Gamma_{0}}$ & $9.0460 \mathrm{E}-02$ & $2.6825 \mathrm{E}-02$ & $7.1011 \mathrm{E}-03$ & $1.8020 \mathrm{E}-03$ & $4.5188 \mathrm{E}-04$ & $1.1302 \mathrm{E}-04$ \\
order & & 1.7537 & 1.9175 & 1.9785 & 1.9956 & 1.9995 \\
$\left\|u-u_{N}\right\|_{\frac{1}{2}, *, \Gamma_{0}}$ & $8.4367 \mathrm{E}-02$ & $2.5019 \mathrm{E}-02$ & $6.6220 \mathrm{E}-03$ & $1.6799 \mathrm{E}-03$ & $4.2110 \mathrm{E}-04$ & $1.0522 \mathrm{E}-04$ \\
order & & 1.7537 & 1.9177 & 1.9788 & 1.9959 & 1.9997 \\
\hline
\end{tabular}

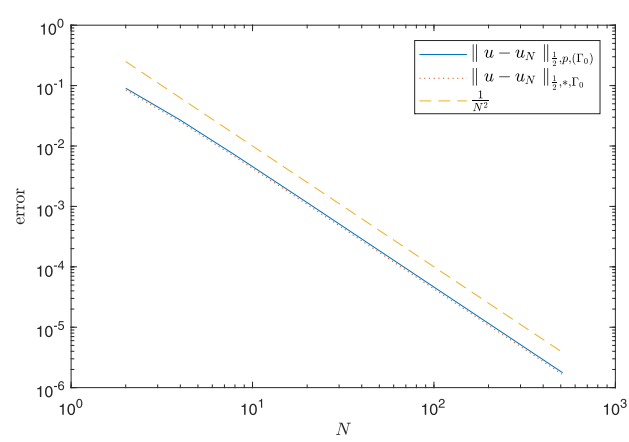

Figure 2 Numerical convergence results with respect to $N$ in Example 1
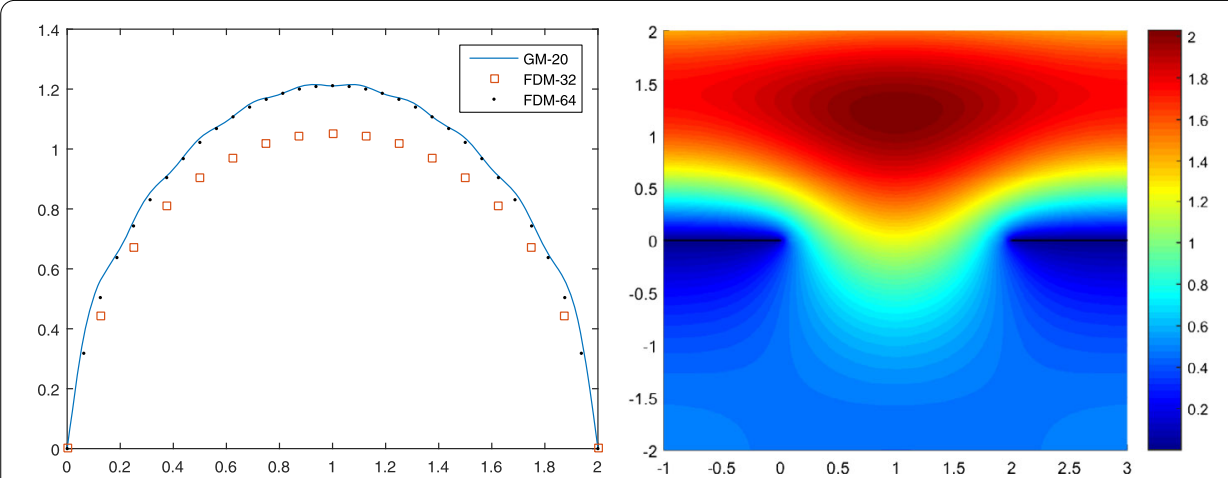

Figure 3 Example 2. (left) Absolute value of total field $u_{t}$ on $\Gamma_{0}$, (right) Absolute value of total field $u_{t}$

so we have $u\left(x_{1}\right) \in H_{p}^{\frac{5}{2}-\varepsilon}\left(\Gamma_{0}\right)$ with $\varepsilon>0$ arbitrarily small. Results are presented in Table 1 and Fig. 2. From these results, we can see that the errors $\left\|u-u_{N}\right\|_{\frac{1}{2}, *, \Gamma_{0}}$ and $\left\|u-u_{N}\right\|_{\frac{1}{2}, p, \Gamma_{0}}$ decay rapidly with respect to $N$, and the corresponding convergence orders are coincident with Theorem 5.

Example 2 We consider the situation of small wave number. Let the wave number $k=1$, and the dimension of $V_{N}$ be $N=10$. The incident wave is plane wave with the incident angle $\theta=0$. Results are shown in Figs. 3-4. In Fig. 3, we show the absolute value of the total field $u_{t}$ on $\Gamma_{0}$ and the total field $u_{t}$ in one period. In Fig. 4, we show the real part and the imaginary part of total field $u_{t}$. From these results we can see that our method is effective when the wave number $k$ is small. Further, in the left one of Fig. 3, we also show the results given by FDM (finite difference method). We demonstrate two results given by FDM with 32 and 64 nodes in one period. Comparing with the FDM, we can see the 

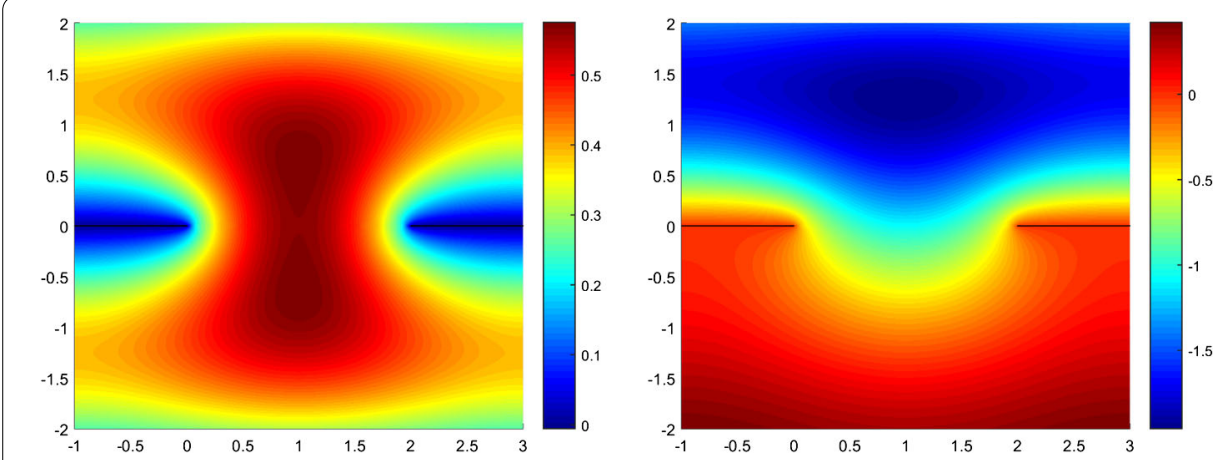

Figure 4 Example 2. (left) Real part of total field $u_{t}$, (right) Imaginary part of total field $u_{t}$
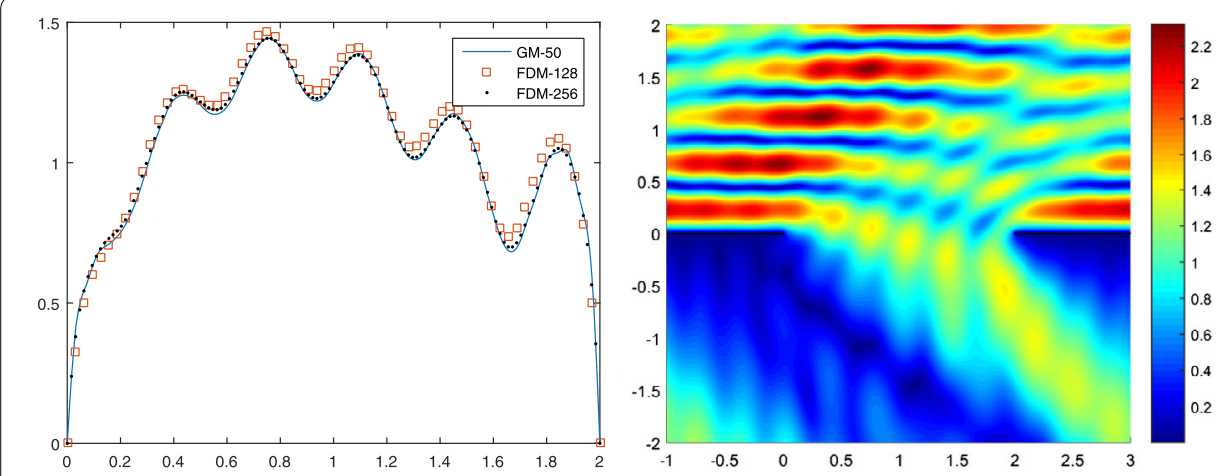

Figure 5 Example 3. (left) Absolute value of total field $u_{t}$ on $\Gamma_{0}$, (right) Absolute value of total field $u_{t}$

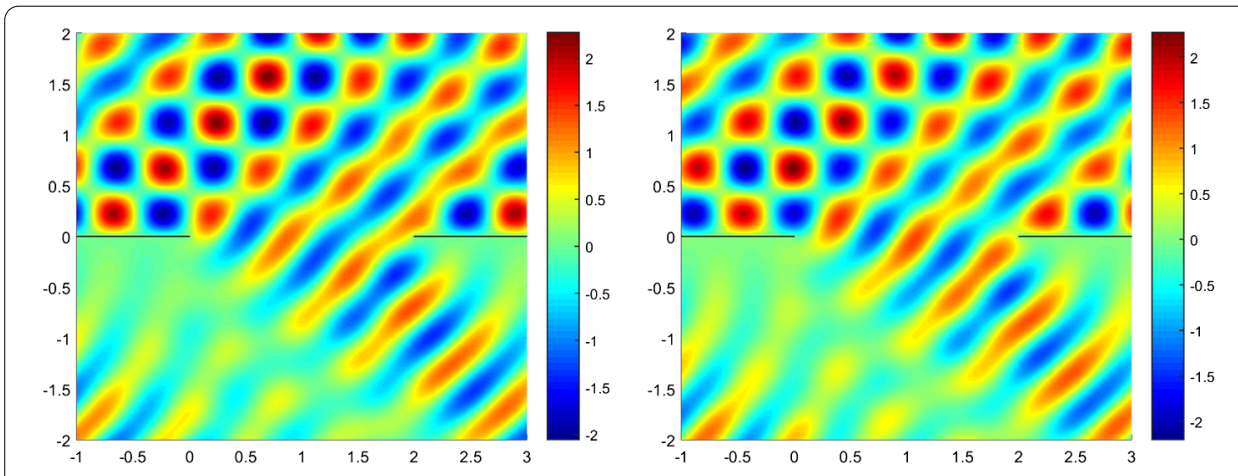

Figure 6 Example 3. (left) Real part of total field $u_{t}$, (right) Imaginary part of total field $u_{t}$

results of our Galerkin method with 20 dofs (degree of freedoms) are coincident with the results of FDM with 64 dofs, i.e., our Galerkin method needs fewer dofs than the FDM.

Example 3 We consider the case of wave number $k=10$. The dimension of $V_{N}$ is $N=50$. The incident wave is plane wave with the incident angle $\theta=\pi / 4$. Results are presented in Figs. 5-6. From these results, we can see that the Galerkin method works well and needs fewer dofs than the FDM to obtain reliable results. 

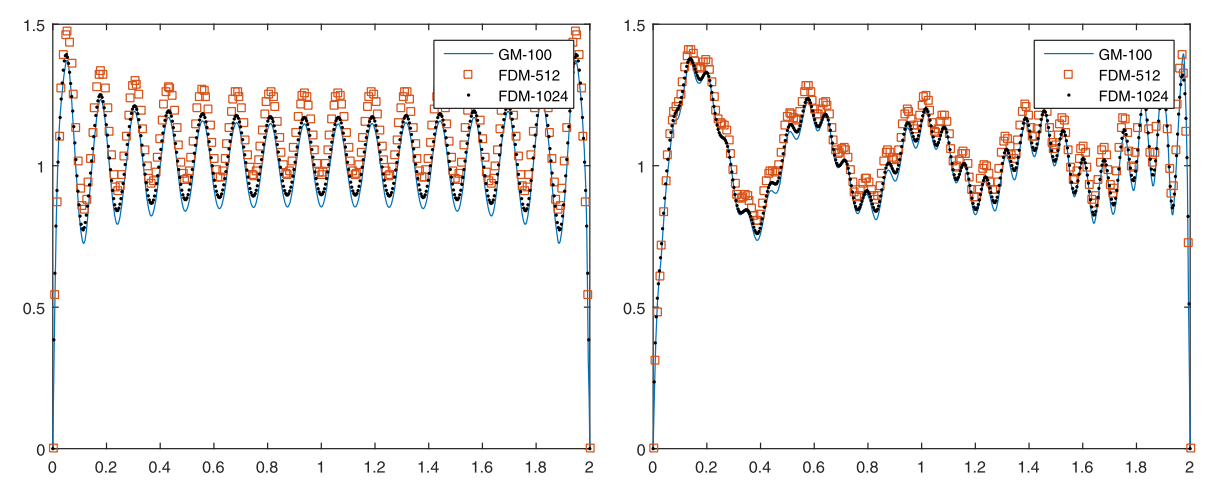

Figure 7 Example 4. (left) Absolute value of total field $u_{t}$ on $\Gamma_{0}$ with $k=50$ and $\theta=0$, (right) Absolute value of total field $u_{t}$ on $\Gamma_{0}$ with $k=50$ and $\theta=\pi / 4$
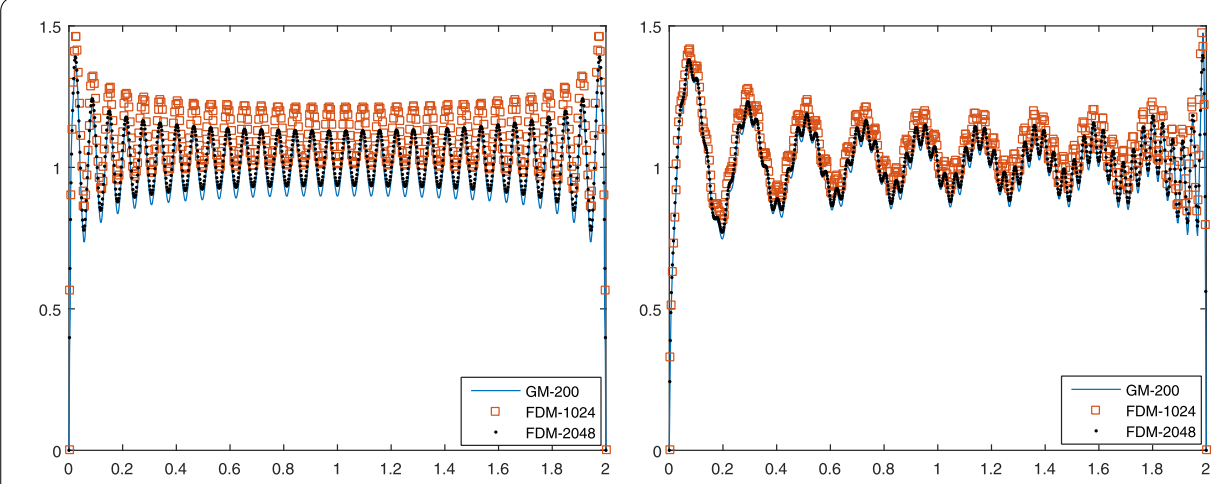

Figure 8 Example 4. (left) Absolute value of total field $u_{t}$ on $\Gamma_{0}$ with $k=100$ and $\theta=0$, (right) Absolute value of total field $u_{t}$ on $\Gamma_{0}$ with $k=100$ and $\theta=\pi / 4$

Example 4 In this example, we consider the situation of large wave number $k=50$ and 100. For $k=50$, we set $N=100$. The absolute values of total fields $u_{t}$ on $\Gamma_{0}$ are presented in Fig. 7 with incident angles $\theta=0$ and $\theta=\pi / 4$, respectively. For $k=100$, we set $N=200$. The absolute values of total fields $u_{t}$ on $\Gamma_{0}$ are presented in Fig. 8 with incident angles $\theta=0$ and $\theta=\pi / 4$, respectively. Also the numerical solutions given by the FDM are shown in these figures. When $k=50$, compared with the FDM which needs 1024 dofs, our Galerkin method needs only 100 dofs to get reliable computational results. When $k=100$, the FDM needs 2048 dofs, while our Galerkin method needs only 200 dofs.

\section{Conclusion}

In this paper, we study the scattering problem of strip gratings. By use of the continuity of the total field across the slit in one period and the Dirichlet to Neumann map, this problem is reformulated to an operator equation on the slit. The well-posedness of the solution to the operator equation is proved and Galerkin method is employed to solve this operator equation. We also derive the error estimate for the Galerkin method and numerical examples show that our method is effective. 


\section{Funding}

This work is supported by the Fundamental Research Funds for the Central Universities (Grant no. 3132017053, 3132018226), the Doctoral Scientific Research Foundation of Liaoning Province (Grant no. 20170520289), TianYuan Special Funds of the National Natural Science Foundation of China (Grant no. 11626054), and the National Natural Science Foundation of China (Grant no. 11601056).

\section{Abbreviations}

MoM, method of moments; FMM, Fourier modal method; CBCM, combined boundary conditions method; FDM, finite difference method; FEM, finite element method; DtN, Dirichlet to Neumann; dofs, degree of freedoms.

\section{Availability of data and materials}

Not applicable.

\section{Competing interests}

The authors declare that they have no competing interests.

\section{Authors' contributions}

The main idea of this paper was proposed by EZ, the theoretical analysis was carried out by the two authors together, and the numerical experiments were conducted by EZ. Both authors read and approved the final manuscript.

\section{Publisher's Note}

Springer Nature remains neutral with regard to jurisdictional claims in published maps and institutional affiliations.

Received: 9 August 2018 Accepted: 29 January 2019 Published online: 13 February 2019

\section{References}

1. Petit, R.: Electromagnetic Theory of Gratings, vol. 22. Springer, Heidelberg (1980)

2. Bao, G., Cowsar, L., Masters, W.: Mathematical Modeling in Optical Science, vol. 22. SIAM, Philadelphia (2001)

3. Wilcox, C.H.: Scattering Theory for Diffraction Gratings. Applied Mathematical Sciences Ser., vol. 46. Springer, New York (1984)

4. Uchida, K., Nora, T., Matsunaga, T.: Electromagnetic wave scattering by an infinite plane metallic grating in case of oblique incidence and arbitrary polarization. IEEE Trans. Antennas Propag. 36(3), 415-422 (1988)

5. Wu, T.K.: Fast converging integral equation solution of strip gratings on dielectric substrate. IEEE Trans. Antennas Propag. 35(2), 205-207 (1987)

6. Lee, C.W., Son, H.: Analysis of electromagnetic scattering by periodic strip grating on a grounded dielectric/magnetic slab for arbitrary plane wave incidence case. IEEE Trans. Antennas Propag. 47(9), 1386-1392 (1999)

7. Florencio, R., Boix, R.R., Encinar, J.A.: Enhanced MoM analysis of the scattering by periodic strip gratings in multilayered substrates. IEEE Trans. Antennas Propag. 61(10), 5088-5099 (2013)

8. Matsushima, A., Itakura, T.: Singular integral equation approach to plane wave diffraction by an infinite strip grating at oblique incidence. J. Electromagn. Waves Appl. 4(6), 505-519 (1990)

9. Montiel, F., Neviere, M.: Electromagnetic study of the diffraction of light by a mask used in photolithography. Opt. Commun. 101(3-4), 151-156 (1993)

10. Montiel, F., Neviere, M.: Perfectly conducting gratings: a new approach using infinitely thin strips. Opt. Commun. 144(1-3), 82-88 (1997)

11. Guizal, B., Felbacq, D.: Electromagnetic beam diffraction by a finite strip grating. Opt. Commun. 165(1-3), 1-6 (1999)

12. Granet, G., Guizal, B.: Analysis of strip gratings using a parametric modal method by Fourier expansions. Opt. Commun. 255(1-3), 1-11 (2005)

13. Elamine, H., Guizal, B., Oueslati, M., Gharbi, T.: New reformulation of the Fourier modal method with spatial adaptive resolution for multilayered metallic strip grating. Opt. Commun. 283(21), 4392-4396 (2010)

14. Li, L.: Fourier modal method. In: Popov, E. (ed.) Gratings: Theory and Numeric Applications, 2nd edn., pp. 1-40. Aix Marseille Université, Marseille (2014)

15. Wang, Y., Ma, F., Zheng, E.: Galerkin method for the scattering problem of a slit. J. Sci. Comput. 70(1), 1-18 (2017)

16. Chandler-Wilde, S.N., Hewett, D.P., Moiola, A.: Interpolation of Hilbert and Sobolev spaces: quantitative estimates and counterexamples. Mathematika 61(2), 414-443 (2015) 